\title{
La documentación audiovisual en las empresas audiovisuales locales e instituciones universitarias andaluzas
}

\author{
María Ángeles López Hernández \\ María Dolores Rodríguez Brito \\ Universidad de Sevilla, Departamento de Periodismo
}

\subsection{Resumen}

Estado actual del proyecto, desarrollado por el Grupo de Investigación en Comunicación Científica y Desarrollo del Departamento de Periodismo de la Universidad de Sevilla, sobre la situación real de la práctica documental en las empresas audiovisuales de la Comunidad Autónoma Andaluza.

Palabras clave: Archivos audiovisuales. Andalucía. Empresas audiovisuales.

\subsection{Abstract}

State of the proyect, developed by the Research Group in Scientific Communication and Development of the Departament of Journalism of the University of Sevilla, on the current real situation of the documentary practice in the audiovisual organizations of Andalucía.

Keywords: Audiovisual archives. Audiovisual companies. Andalusia (Spain).

\section{Introducción}

Hoy en día no resulta ya necesario defender, puesto que es por todos conocida, la importancia e interés que reviste para cualquier empresa o institución, incluida naturalmente la que produce o conserva material audiovisual, el contar con un centro de documentación que gestione y regule la difusión de los documentos generados por ella (documentos propios) y los procedentes de fuentes ajenas. La acumulación de experiencias demuestra y certifica cada vez más claramente que poseer este tipo de centros es altamente rentable, y en los foros nacionales e internacionales se habla de la conveniencia de ponerlos en marcha en todas las organizaciones, tanto en las antiguas que aún no cuentan con este departamento como en las nuevas que deben integrarlo originariamente en su organigrama. La expansión —o más propiamente explosión- de nuevas fuentes 
de información como Internet ha contribuido grandemente a esta concienciación social de la relevancia de contar con un equipo de documentalistas que controlen el gran volumen de documentos que circulan dentro y fuera de la organización.

En el ámbito de las empresas privadas (caso de las televisiones y productoras) y de las instituciones públicas (como las universitarias), la práctica documental se ha ido asentando en la última década, siendo ya casi impensable el que una organización no cuente con un servicio de documentación dentro de su organigrama. Cada vez son más los que defienden la tesis de que una organización que no controla y gestiona adecuadamente la información (siguiendo para ello técnicas documentales), difícilmente puede ofrecer a su audiencia - en el caso de la televisión — o a sus lectores — en el caso de la prensa — o a sus usuarios investigadores - en el caso de las universidades - un producto de calidad. No obstante, esta realidad sólo se hace patente en el seno de las grandes organizaciones, pero se desconoce si la documentación está calando con la misma fuerza en las pequeñas y medianas empresas audiovisuales y en las instituciones públicas que no cuentan con un amplio u holgado presupuesto económico. Por esta razón, el Grupo de Investigación en Comunicación Científica y Desarrollo del Departamento de Periodismo de la Universidad de Sevilla, decidió emprender un trabajo de campo (1) para conocer la situación por la que atravesaba la práctica de la Documentación en la Comunidad Autónoma Andaluza. El trabajo que aquí presentamos es, pues, el resumen de una investigación iniciada hace ya más de un año y que, dada su envergadura, aún no ha sido concluida. Pero vayamos paso a paso, explicando con detalle el proyecto y los resultados que hasta el momento se han obtenido.

\section{Objetivos y alcance de la investigación}

El fin perseguido con este proyecto de investigación es, en esencia, conocer, por un lado, la práctica documental desarrollada en los medios audiovisuales andaluces (televisiones locales y productoras), así como la política audiovisual seguida en las instituciones universitarias de nuestra Comunidad, al objeto de: en primer lugar, elaborar un censo, lo más completo posible, de los servicios de documentación audiovisuales existentes en Andalucía; en segundo, hacer un estudio analítico, a partir de los datos recopilados, de las tareas desarrolladas por los distintos centros de documentación investigados, a fin de conocer el estado y desarrollo práctico de la documentación en los medios audiovisuales e instituciones universitarias de la Comunidad Autónoma Andaluza.

Aunque nuestro propósito es el de extender el estudio a toda Andalucía, se ha comenzado, en una primera fase, por conocer la situación de los sistemas de información audiovisuales en las provincias de Andalucía Occidental: Cádiz, Córdoba, Huelva y Sevilla. A fin de que el trabajo que emprendimos fuera lo más representativo posible de la realidad documental en Andalucía, se hizo un exten-

Scire. $8: 2$ (jul.-dic. 2002) 159-172. 
so barrido por los siguientes medios audiovisuales: productoras, televisiones locales y videotecas adscritas a las universidades públicas de nuestra Comunidad Autónoma. Se dejaron a un lado los medios televisivos públicos (Canal Sur y Centro Territorial de TVE), cuya actividad documental ya nos es conocida.

\section{Fases de investigación y metodología de trabajo}

La investigación se ha desarrollado en varias etapas. En primer lugar, se elaboró un censo de las productoras, las televisiones locales y las videotecas universitarias existentes en Cádiz, Córdoba, Huelva y Sevilla. La metodología utilizada para localizar dichas organizaciones consistió en: a) el conocimiento directo que los propios miembros del grupo de investigación tenían de algunas de ellas; b) el empleo de ciertas fuentes de información, como diversas guías, directorios e Internet; c) los contactos telefónicos o personales con instituciones (por ejemplo, ayuntamientos) y con algunos medios que nos proporcionaron, a su vez, siguiendo una cadena, información sobre otras organizaciones audiovisuales de su entorno.

En segundo lugar, al objeto de recopilar la información que nos era precisa para nuestro censo, decidimos, en principio, entablar contacto con las empresas audiovisuales enviándoles un cuestionario — que más tarde describiremos- por correo electrónico, al considerar este medio como más rápido y práctico. No obstante, ante la falta absoluta de respuesta (sólo nos remitieron un cuestionario y sin rellenar), consideramos que lo más práctico era contactar directamente con tales empresas mediante llamadas telefónicas, consiguiendo en esta ocasión mejores resultados, no sólo ya por los cuestionarios que pudieron rellenarse, sino también porque gracias a dichas llamadas pudimos saber que muchas de las empresas figurantes en nuestra base de datos ya no existían, habían cambiado de nombre o de domicilio social. Finalmente, y dado que tras sucesivos intentos no pudimos contactar con ciertas empresas audiovisuales o con los encargados de sus archivos, decidimos remitirles a éstas, al igual que habíamos hecho antes con las instituciones universitarias (de las que obtuvimos rápida contestación), el cuestionario por correo ordinario, intentando asegurarnos la respuesta mediante la inclusión, dentro del sobre enviado, de otro sobre con nuestra dirección y el sello de correos. Esta táctica, aun cuando parece dar resultados, es sumamente lenta, hasta el punto de que, tras haber transcurrido varios meses, todavía se están recibiendo los últimos cuestionarios. Permítasenos decir, en vista de lo comentado, que ésta ha sido una tarea ingente, que nos ha llevado mucho tiempo, mucho esfuerzo, y que en ocasiones ha resultado desalentadora por la escasa participación y la falta de compromiso de muchas empresas — no así de los centros universitarios- que veían con recelo nuestra investigación.

En tercer lugar, partiendo de los resultados obtenidos en nuestra investigación, uno de nuestros próximos proyectos consistiría en una doble tarea: prime-

Scire. $8: 2$ (jul.-dic. 2002) 159-172. 
ro, en el diseño de un modelo de sistema de información aplicable tanto a los medios audiovisuales (productoras, televisiones locales, etc.) desabastecidos de servicio documental, como a las instituciones universitarias carentes de videotecas independientes; $y$, segundo, en la instrucción acerca de la aplicabilidad del modelo diseñado en aquellas organizaciones que lo demandaran.

En el trabajo de campo se les pidió a los medios audiovisuales y centros universitarios con los que se contactó que respondieran o rellenaran un cuestionario (figura 1), el cual nos proporcionaría los datos que nos eran necesarios para cubrir los objetivos informativos de nuestra investigación. Las preguntas formuladas en el cuestionario se dividen en tres grupos: por un lado, las que hacen referencia al tipo de documentos conservados, permitiéndonos valorar la riqueza documental de la organización (preguntas 1, 2 y 3); y, por otro, las que hacen alusión a si la empresa o institución cuenta con centro de documentación o archivo y, en caso

1. ¿Qué tipo de documentos se conserva en su organización?
o Periódicos
o Revistas especializadas
o Folletos
o Informes y proyectos
o Material audiovisual:
o Vídeo:
Tipo/s de formato:
o Película:
Tipo/s de formato:
o Grabaciones sonoras
o Fotografías

2. De esos documentos, ¿cuáles son de producción propia y cuáles de producción ajena?

- Documentos de producción propia:

- Documentos de producción ajena:

3. Los materiales audiovisuales conservados, ¿en torno a qué contenidos giran?:

o Programas de entretenimiento (concursos, magazines...)

o Programas informativos (reportajes, entrevistas, noticias...)

o Programas deportivos

o Documentales

o Documentos publicitarios

4. ¿Cuenta su organización con un servicio de archivo o de documentación?
— En caso afirmativo:

5. ¿Cuántas personas están encargadas de dicho servicio?

6. ¿El servicio de documentación proporciona información sólo a los trabajadores de la empresa o también a usuarios externos (investigadores, profesionales, etc.)?

7. ¿Qué tratamiento reciben esos documentos?

— Producción propia:

o Registro

o Descripción física y/o catalogación

o Resumen

o Indización por palabras claves

o Automatización (especificar el programa de gestión de información empleado)

- Producción ajena:

o Registro

o Descripción física y/o catalogación

o Resumen

o Indización por palabras claves

o Automatización (especificar el programa de gestión de información empleado)

8. ¿Mantiene el centro de documentación alguna política de intercambio con otras instituciones? ¿Podría precisar con cuáles?

Figura 1: Cuestionario remitido a los medios audiovisuales de Andalucía

Scire. $8: 2$ (jul.-dic. 2002) 159-172. 
afirmativo, el tipo de tratamiento documental que éstos dan a los documentos conservados (preguntas 4, 5 y 7). Ello nos permitirá dibujar un mapa general de la irrupción de la Documentación Audiovisual en las pequeñas y medianas organizaciones audiovisuales andaluzas y, por derivación, calibrar la difusión e interés que esta disciplina científico-técnica tiene en el marco de nuestra comunidad autónoma. Las preguntas 6 y 8 tenían como finalidad conocer la posibilidad o grado de accesibilidad a la información por parte de usuarios externos a la organización y la política de intercambios que mantienen entre sí tales organizaciones.

Con la información que se ha ido recogiendo se ha elaborado una base de datos (2) (utilizando el programa de gestión de bases de datos Wknosys) en la que se especifican los siguientes campos: nombre de la organización, tipo de organización - productora de vídeo o televisión, televisión local, institución universitaria, etc.-, provincia, dirección, dirección electrónica, teléfono, fax, departamento - en este campo se consigna si la organización cuenta o no con servicio de documentación o con archivo-, tareas documentales — se especifica el tipo de tratamiento al que se someten los documentos conservados en la organización: registro, descripción, análisis e indización, etc.—, y otros datos.

\section{Resultados y discusión}

Antes de iniciar este apartado dedicado a los resultados hemos de advertir que, de los aproximadamente ciento veinticinco formularios o encuestas realizadas, hemos conseguido reunir hasta el momento setenta y una, de las cuales cuatro corresponden a las universidades andaluzas (Cádiz, Córdoba, Huelva y Sevilla), y sesenta y siete a televisiones locales y productoras, una cifra nada desdeñable teniendo en cuenta los recelos de muchas empresas e instituciones a contestar cuestionarios, aun teniendo éstos una finalidad puramente investigadora como les hacíamos saber de antemano. Además, desde nuestra óptica, esta cifra resulta suficientemente significativa a la hora de dibujar el panorama general en el que se desenvuelve la Documentación Audiovisual en nuestra Comunidad Autónoma.

Para una más clara comprensión de los resultados obtenidos, iremos tratando separadamente cada una de las preguntas formuladas en el cuestionario, dando a conocer, primero, los datos porcentuales y, segundo, efectuando una posible lectura de éstos. Hemos de decir además que, dadas las características propias de las instituciones universitarias, se ha decidido analizarlas independientemente del resto de los medios productores de material audiovisual, sin que ello reste significación al estudio; entre otros motivos, porque contamos con los datos de las cuatro universidades, ubicadas en Andalucía Occidental, que han sido encuestadas.

Así pues, y por lo que respecta a la primera pregunta enunciada - ¿Qué tipo de documentos conserva en su organización? - los resultados indican que, de las

Scire. $8: 2$ (jul.-dic. 2002) 159-172. 
65 empresas audiovisuales encuestadas, sólo un $15 \%$ posee otras fuentes de información distintas a las videográficas, siendo el porcentaje más amplio el correspondiente a las grabaciones sonoras. Por su parte, y como era de esperar al tratarse de medios audiovisuales, el 84,6\% posee material videográfico en diferentes formatos, siendo lo más común el contar con un único tipo de soporte (el $58,2 \%)$ y rara vez con tres o más de tres $(20 \%)$. Entre los sistemas de reproducción más empleados se encuentran el Super-VHS (45, 4\%), el Betacam $(41,8 \%)$ y el VHS $(32,7 \%)$, mientras que otros sistemas más antiguos, retirados del mercado, caso por ejemplo del U-Matic, sólo alcanzan el porcentaje de 5,4\%. Los nuevos soportes digitales no superan el 18\%, no habiendo, en este último caso, apenas homogeneidad en los sistemas empleados por una y otra empresa. De todo ello podemos deducir, en primer lugar, la escasa riqueza de los fondos documentales que poseen estas empresas audiovisuales, la mayoría de las cuales se nutren únicamente de la producción videográfica, lo que nos da a entender que utilizan muy pocas fuentes para la elaboración de su trabajo o, dicho en otros términos, que hay una exigua labor investigadora previa para preparar y producir los nuevos documentos audiovisuales que generan. En segundo lugar, es de destacar el uso habitual de un solo soporte o formato videográfico, siendo los más extendidos, como hemos visto, los formatos profesionales Super-VHS y Betacam y el soporte no profesional VHS. Los antiguos soportes son prácticamente inexistentes en la mayoría de los medios audiovisuales andaluces, lo que puede deberse al moderno origen de muchos de estos medios, los cuales no han tenido siquiera oportunidad de utilizarlos al haber desaparecido, antes de comenzar su singladura, del mercado. Por último, y en lo que respecta a los sistemas de reproducción digitales, cabe destacar que, aun cuando no están suficientemente extendidos, comienzan a entrar con fuerza en el mercado audiovisual andaluz. No obstante, la nota predominante en este caso es la gran dispersión o diversidad de los soportes digitales utilizados por uno y otro medio; algo que debe tenerse muy en cuenta debido a que el futuro digital no está todavía resuelto. Esto hace presuponer que algunos de los soportes empleados en la actualidad queden obsoletos o desaparezcan del mercado en poco tiempo, realidad ésta que no sabemos hasta que punto han sabido calibrar suficientemente las empresas audiovisuales. En lo referente al ámbito universitario se ha de decir que las cuatro universidades andaluzas encuestadas poseen, aparte naturalmente de documentos bibliográficos, fondos videográficos en sus bibliotecas o en sus videotecas, además de otras fuentes de información primarias también relevantes para la organización y sus usuarios, como publicaciones periódicas, folletos, informes, proyectos o grabaciones sonoras, fuentes éstas que suponen en algunos casos hasta el $20 \%$ del total de los fondos. En cuanto al tipo de soporte videográfico conservado en estas instituciones, sólo una de ellas posee más de un formato, siendo el sistema habitual 
el VHS doméstico, aunque puede observarse hoy ya una tendencia paulatina a la utilización de los nuevos soportes digitales.

De estos datos relativos a las universidades podemos inferir algunas conclusiones importantes. En primer lugar, existen fondos videográficos en todos los centros universitarios. En segundo lugar, los fondos audiovisuales constituyen un porcentaje muy inferior a los fondos bibliográficos. Y, en tercer lugar, en repuesta a las demandas informativas de los usuarios, la mayor parte de la colección videográfica se encuentra en formato doméstico VHS, al ser éste el sistema más extendido en los vídeos domésticos. De esto último podemos derivar, a su vez, la hipótesis de que a medida que los nuevos sistemas de reproducción digitales entren en los hogares, también entrarán en las universidades.

Con respecto a la segunda pregunta formulada en el cuestionario - De los documentos conservados en la organización ¿cuáles son de producción propia y cuáles de producción ajena?-, los datos indican que el 67,7\% de las televisiones locales y de las productoras andaluzas sólo conserva documentos de producción propia, mientras que el 24,6\% también guarda otras fuentes de producción ajenas, siendo las más frecuentes revistas especializadas, periódicos, publicidad, grabaciones sonoras y documentos videográficos comprados o cedidos por televisiones locales asociadas. El resto de los medios, el 7,7\%, afirma no conservar ni producción ajena ni aun siquiera producción propia, ya que, según éstos, no pueden hacer uso de la misma al haber vendido sus derechos a otras organizaciones. No obstante, pensamos que, a pesar de su afirmación, lo más probable es que conserven los documentos propios, aunque, por razones legales, no quieran hacérnoslo saber. Estos resultados porcentuales nos confirman en nuestra hipótesis de partida, según la cual la mayoría de las empresas privadas audiovisuales priman la selección de los documentos generados por ellas antes que los procedentes de organizaciones ajenas; lo que, por otro lado, es comprensible, ya que si cada uno no custodia sus documentos difícilmente lo hará por él otra organización. Por tanto, vemos cómo los fondos documentales de las televisiones locales y las productoras se nutren esencialmente de la producción propia. No obstante, sabemos - y tales medios deberían saberlo también — que dichos documentos no son por sí mismos suficientes para cubrir de manera efectiva todas las necesidades informativas de la organización: el archivo debería contar con una más amplia gama de fuentes, ya sean éstas textuales (monografías, obras de referencia, folletos, recortes de noticias, etc.), visuales (fotografías) y/o sonoras (CD, discos, casetes, etc.), cuyos contenidos temáticos sirvan para enriquecer la colección del centro y facilitar la labor de investigación de sus usuarios. En el terreno universitario, los datos relativos a esta pregunta sobre producción propia o ajena varían radicalmente de los obtenidos de las televisiones locales y las productoras. Así, los datos indican que el $80 \%$ de las universidades encuestadas conservan

Scire. 8 : 2 (jul.-dic. 2002) 159-172. 
únicamente documentos de producción ajena y tan sólo un $20 \%$ también posee producción propia entre sus fondos. Ello se debe a que las instituciones universitarias adquieren los fondos videográficos como apoyo a las líneas de docencia e investigación que se desarrollan en su seno.

Siguiendo adelante, y en lo que respecta a la tercera cuestión — Los materiales audiovisuales conservados ¿en torno a qué contenidos giran?-, los datos estadísticos muestran, primeramente, que la mayoría de los medios audiovisuales encuestados no están especializados en la producción de un tipo concreto de programa, sino que por lo general cultivan varias categorías distintas, llegando incluso el $33,8 \%$ de estos medios a elaborar todo tipo de programas. Los documentos más numerosos, de entre los producidos por las empresas audiovisuales andaluzas, son los categorizados como informativos (reportajes, entrevistas, noticiarios, documentales...), siguiéndoles de cerca los programas de entretenimiento, mientras que tan sólo entre el 10\% y el 16\% de los medios producen programas deportivos o publicitarios, y en torno al 6,1\% documentos institucionales y videoclips musicales. En el caso de los fondos videográficos universitarios, éstos se componen esencialmente de programas educativos y divulgativos (que suponen el 75\%), como, por ejemplo, documentales, entrevistas, reportajes, obras cinematográficas..., en definiva, documentos todos ellos que guardan estrecha relación con las diversas materias que se imparten en los centros universitarios.

Hasta aquí hemos analizado el primer bloque de preguntas contenidas en el cuestionario, todas ellas relativas a la naturaleza de los documentos guardados o custodiados en la organización. A partir de ahora entraremos en un segundo bloque de cuestiones que aluden a la actividad puramente documental que desarrolla o no dicha organización. La primera pregunta de este segundo bloque $-i$ Cuenta su organización con un servicio de archivo o de documentación? - va a ser analizada junto a otras dos cuestiones, en nuestra opinión, íntimamente relacionadas con ella, cuales son: ¿Cuántas personas están encargadas de dicho servicio? y ¿Qué tratamiento reciben esos documentos? Dicha interrelación establecida por nosotros entre las tres cuestiones se explica por el hecho de que muchas de las empresas audiovisuales consultadas — no así las instituciones universitarias- no tenían claro "a qué nos referíamos cuando hablábamos de centro de documentación", con lo que su respuesta no nos resultaba absolutamente fiable. Entonces decidimos utilizar las dos preguntas siguientes, antes citadas, para establecer los límites entre aquellas empresas qué contaban con archivo y las que contaban con centro de documentación. Así, primeramente, nos fijamos en el tipo de tratamiento documental que la organización daba a los documentos conservados, considerando que: a) si no existía tratamiento documental alguno, hablaríamos de almacenamiento, ya que al fin y al cabo lo único que tales organizaciones hacen con sus documentos es colocarlos en una estantería; b) si los documentos eran registrados,

Scire. $8: 2$ (jul.-dic. 2002) 159-172. 
descritos físicamente o se contaba con una base de datos, lo que supone cierto nivel de control de la información ingresada, entonces hablaríamos de archivo, puesto que los documentos no sólo son almacenados sino que, además, se arbitra alguna fórmula para su localización en los estantes; c) finalmente, si los documentos eran registrados, descritos físicamente, ingresados en una base de datos y sometidos a cierto análisis documental —ya fuera resumen, indización o ambos-, hablaríamos de centro de documentación. También la pregunta relativa al número de personas encargadas del servicio de documentación resulta reveladora para conocer si la organización cuenta o no con archivo o con centro de documentación. Así, por ejemplo, es significativo que aquellas organizaciones que habíamos categorizado de "sólo almacenamiento", respondían que nadie se hacía cargo "normalmente" de este servicio, mientras que, en el lado opuesto, las consideradas por nosotros como empresas con "centro de documentación" decían contar, por línea general, con dos o tres personas encargadas de dicho servicio de documentación. Pero pasemos a analizar con más detenimiento estos datos esbozados.

Los resultados porcentuales obtenidos nos muestran que el 53,8\% de los medios audiovisuales andaluces encuestados poseen un archivo, el 29,2\% cuenta con un centro de documentación y, finalmente, el 17\% sólo almacena los documentos. A su vez, de aquellos medios que cuentan con archivo o centro de documentación, el 38,9\% responde que sólo tiene una persona encargada de este servicio; el 29,6\% manifiesta que nadie en particular dentro de la organización se hace cargo de este servicio; el 24\% sostiene que cuenta con dos personas; y sólo el 7,4\% dice tener tres o más personas dedicadas a la gestión de los documentos. El que haya más archivos que centros de documentación en los medios audiovisuales andaluces, unido al hecho de que la mayoría de ellos cuenten con una sola persona o con "nadie en particular" para gestionar los fondos, nos hace inferir la escasa importancia que aún se le otorga a la práctica documental en estas organizaciones, una situación que puede deberse, en nuestra opinión, a dos factores básicos, posiblemente interrelacionados, como son: el económico y el de la falta de información, por parte de dichos medios, acerca de los objetivos y funciones de un servicio de documentación. Detengámonos algo más en ambos factores. Respecto al factor económico, todos somos conscientes de que la puesta en marcha de un servicio de documentación no es nunca una tarea fácil ni barata. Son muchos los aspectos que se han de contemplar y calibrar convenientemente cuando se proyecta un centro de documentación, desde su planificación física (localización, mobiliario, equipamiento técnico informático, software de gestión de información...), pasando por el análisis de las necesidades informativas de la organización, hasta concluir, incluso, con la redacción de un manual de funcionamiento interno. Ello supone, qué duda cabe, una fuerte inversión económica, inversión que la mayoría de las medianas o pequeñas organizaciones audiovisuales que nacen en el ámbito

Scire. $8: 2$ (jul.-dic. 2002) 159-172. 
local o provincial no puede afrontar o, lo que es peor aún, no quiere afrontar, ya que desconoce las ventajas y los beneficios que a medio o largo plazo puede llegar a proporcionarles el centro de documentación. Precisamente, relacionado con este último comentario se encuentra el segundo factor al que antes aludíamos. Nos referimos a la falta de información que las empresas audiovisuales poseen acerca de lo que es un centro de documentación y del interés que puede revestir para ellas el contar con un servicio de información bien organizado. En este caso, la culpa parece estar en nosotros mismos, es decir, en quienes nos dedicamos al estudio y a la práctica de la Documentación Audiovisual, que no hemos sido capaces de transmitir a las empresas, para las que supuestamente debemos trabajar, nuestro más fiel convencimiento acerca de la importancia que reviste la Documentación en nuestra moderna sociedad, donde el flujo de información se intensifica y se especializa, siendo necesario su control y manejo por personas expertas. Esta situación de insuficiencia documental que se da en las televisiones y productoras no sucede en la esfera universitaria, donde los datos recopilados constatan que el 100\% de estas instituciones educativas cuentan con archivos perfectamente organizados en los que trabaja personal conocedor de las normas archivísticas y documentales. En estos centros los materiales audiovisuales son registrados, sellados, catalogados, esto es, sometidos a un proceso documental exhaustivo de análisis formal o externo. No obstante, se ha de exponer que de las cuatro universidades encuestadas, sólo una de ellas cuenta con videoteca independiente en algunas de sus facultades, es decir, con un servicio específico para el tratamiento y conservación de los documentos videográficos, mientras que el resto integra estos documentos entre los fondos bibliográficos, esto es, se encuentran localizados en las bibliotecas. Ello puede deberse al hecho de que los materiales audiovisuales no constituyen hoy por hoy una parte esencial de los fondos universitarios, al no estar muy extendida aún la cultura de lo audiovisual en este entorno académico, que sigue sustentado sobre la base tradicional del manejo, consulta y lectura de papel impreso, esto es, de los libros y las revistas. No obstante, no debe pasar desapercibido para estas instituciones que la preservación en estado óptimo de este tipo de documentos audiovisuales requiere de unas condiciones medioambientales y de almacenamiento diferentes a las de los documentos bibliográficos.

En cuanto al tratamiento recibido por los documentos audiovisuales en las televisiones locales y productoras encuestadas, uno de los datos más significativos es el referido a la automatización del servicio de archivo o del centro de documentación. Según los resultados obtenidos, de las 54 empresas audiovisuales que cuentan con un tipo u otro de servicio documental, un total de 34 (el 63\%) trabaja con bases de datos. A su vez, de estos 34 medios, 22 cuentan con archivos y 12 con centros de documentación. Por lo que respecta a las demás empresas audiovisuales, un total de 20, éstas siguen realizando en la actualidad las tareas de forma 
manual, incidiendo esta realidad más sobre los archivos (16) que sobre los centros de documentación, en los que sólo cuatro figuran como no automatizados. Dadas las diferencias palpables que puede darse entre un tipo u otro de servicio documental en las empresas audiovisuales consultadas, pensamos que lo más útil es analizarlos de manera independiente. En los archivos, el nivel de tratamiento difiere de un medio a otro, como puede verse en los siguientes porcentajes. En primer lugar, de los 22 archivos automatizados referidos anteriormente, los que sólo trabajan sobre la base de una hoja de análisis informatizada suponen el 50\%, aunque no se precisa en el cuestionario si los elementos informativos contemplados en dicha ficha de análisis se ajustan o responden a las normas nacionales o internacionales de descripción externa de los documentos. En segundo lugar, los archivos que, además de contar con una hoja de análisis automatizada, registran y/o describen físicamente los documentos suponen el 14,7\%. Finalmente, los archivos que registran y/o describen físicamente los documentos de manera manual suman un total de 16 , lo que supone el $80 \%$ del total de archivos y centros de documentación que aún no poseen una base de datos automatizada. En cuanto a los centros de documentación, los resultados indican, en primer lugar, que de los 12 centros de documentación contabilizados en los cuestionarios reunidos, el 66,7\% trabaja en la actualidad con una base de datos automatizada, mientras que el $33.3 \%$ aun trabaja con fichas manuales de análisis. En segundo lugar, la mayoría de los centros de documentación registran, describen físicamente y resumen los documentos, pero pocos los someten también a un proceso de indización. De hecho, de los 12 centros de documentación estudiados, sólo cinco (el 41,7\%) efectúan un tratamiento documental exhaustivo a los documentos, esto es, los analizan formalmente (registro y descripción física) y documentalmente (resumen e indización). De otro lado, y en lo que respecta a las universidades, todas ellas tienen automatizado su servicio de archivo o biblioteca, trabajando con sistemas de gestión de información integrados, especialmente indicados para el ámbito bibliotecario, que permiten la descripción conjunta del material bibliográfico y del audiovisual. Así, pues, vemos como el material audiovisual recibe el mismo tratamiento documental que las demás fuentes que configuran la colección del centro (libros, revistas, etc.). Un último dato interesante, referido nuevamente a la automatización, y que no queremos pasar por alto es el relativo al tipo de sistema de gestión de información empleado más frecuentemente por los archivos y centros de documentación de las empresas televisivas y de las productoras analizadas. Según los datos reunidos en los cuestionarios, la mayoría de estas empresas privadas (el 70,5\%) emplean un sistema de gestión de información propio, es decir, diseñado a la medida de las necesidades informativas de la organización, mientras que sólo el 17,6\% utiliza el sistema de gestión Access, y el 14,7\% otros tipos diferentes de bases de datos (Office, Dbase IV, Wknosys, etc). Esta dispersión —observable en los sistemas de 
gestión de información empleados y antes también en el análisis documentaldificulta seriamente las posibilidades de intercambio de información entre las organizaciones, lo que se contradice con la paulatina tendencia actual, gracias a Internet, a demoler las barreras que nos alejan de la información.

Pero este hermetismo en el que trabajan las televisiones y productoras audiovisuales andaluzas de carácter local también se hace patente cuando analizamos los resultados porcentuales alcanzados en las dos restantes preguntas del cuestionario, preguntas relativas, por un lado, al tipo de consulta facilitado por la organización - ¿El servicio de documentación proporciona información sólo a nivel interno o también a otros usuarios externos? - y, por otro, al intercambio de información que la organización mantiene con otras empresas o instituciones ¿Mantiene el centro de documentación alguna política de intercambio de información con otras instituciones?. Respecto a la primera pregunta señalada, los datos porcentuales señalan que la mayoría (el 64,6\%) sólo proporciona información a nivel interno, esto es, a los trabajadores de la empresa; las que mantienen una política de información a nivel interno y externo constituyen el 26,1\%, mientras que un 9,2\% no responden (NS/NC) a la cuestión formulada. No obstante, de aquellas que dicen permitir la consulta de sus fondos a usuarios externos, hemos de dejar constancia de que los datos por ellas proporcionados no son del todo fiables, ya que, como estos mismos medios señalan, la consulta de sus fondos está abierta a ciertas instituciones públicas, a otras televisiones asociadas (Acutel o Aprotel: Asociaciones de Televisiones Locales) o a clientes únicamente, con lo que, en realidad, cualquier investigador no tiene tan fácil acceso como se pudiera pensar - o desear - a la información conservada en sus archivos. Se trata, pues, de un servicio de consulta externo, pero restringido. Naturalmente, este dato se invierte cuando hablamos de las universidades, ya que el objetivo esencial de estas instituciones es precisamente el de servir información a los usuarios externos. Aunque es cierto que, de alguna manera, también existe un control de acceso a los documentos, ya que sólo los miembros de la comunidad (alumnos, investigadores, miembros del PAS y profesores) pueden sacarlos en préstamo. Sin embargo, la consulta en sala es libre en la mayoría de los centros universitarios, esto es, cualquier persona, pertenezca o no a este ámbito, puede consultar los fondos del centro. Finalmente, y en lo referente al intercambio de información entre las televisiones y productoras, los datos porcentuales, aun siendo muy similares, muestran no obstante una tendencia mayoritaria hacia la no relación de intercambio, lo que nos confirma, una vez más, en lo antes referido sobre el hermetismo en el que se desenvuelven estas organizaciones. Así, el 53,8\% dice no seguir ningún tipo de intercambio con otras organizaciones, mientras que el $46,1 \%$ restante afirma mantener una política de intercambio con otras productoras o televisiones locales y con instituciones públicas y/o privadas, con las que

Scire. $8: 2$ (jul.-dic. 2002) 159-172. 
guardan, en líneas generales, una relación económica — de clientela—, de asociacionismo o temática, al tratar asuntos informativos comunes. Por otro lado, de las cuatro universidades andaluzas encuestadas, tres dicen mantener relaciones de préstamo interbibliotecario principalmente con otras universidades, sin descartar por ello sus relaciones con otras instituciones.

\section{Conclusiones}

En primer lugar, destaca la escasa riqueza de los fondos documentales que sufre la mayoría de las televisiones y productoras andaluzas de carácter local, lo que se manifiesta tanto en la poca variedad tipológica de los documentos que guardan (la mayoría de las organizaciones sólo cuentan con documentos videográficos), como en el predominio dado a la producción propia frente a la producción ajena, de la que apenas conservan algo en sus fondos ciertas organizaciones, las menos. En cuanto a las universidades, también es constatable la poca importancia otorgada aún a los materiales audiovisuales en estas instituciones, lo que se deduce, primero, del pequeño número que suponen dentro del marco global de las colecciones y, segundo, de que no existan videotecas independientes en tres de las cuatro instituciones consultadas, sino que, por el contrario, los documentos audiovisuales compartan espacio en las estanterías con los libros y las revistas, lo que puede perjudicar grandemente su estado de conservación. Tales documentos, aun cuando existen en todos los centros universitarios, constituyen un punto débil de la colección que debe ser subsanado, sobre todo si tenemos en cuenta que resultan ser un material de apoyo cada vez más importante para las diversas líneas de docencia e investigación desarrolladas en el seno de las universidades.

En segundo lugar, la escasa importancia otorgada en la actualidad a la práctica documental por las televisiones y productoras andaluzas de carácter local, circunstancia ésta que deducimos, por una parte, de que haya más archivos que centros de documentación y, por otra, de que la mayoría de ellos cuenten con una sola persona o con "nadie en particular" para gestionar los fondos. Por lo que respecta a las instituciones universitarias, hemos de decir que en todas ellas los documentos audiovisuales, a pesar de su constatable diferencia con otro tipo de documentos, reciben el mismo tratamiento documental, un tratamiento que se resume, en líneas generales, al análisis formal o externo de sus elementos informativos sustanciales, al objeto de tenerlos localizados en los estantes.

En tercer lugar, el hermetismo en el que desarrollan su actividad profesional la mayoría de las empresas audiovisuales encuestadas, lo que se infiere de numerosos datos recogidos como, por ejemplo: a) la falta de homogeneidad en el tratamiento documental dado a los documentos por uno u otro archivo o centro de documentación; b) la utilización preferente de sistemas de gestión de información creados expresamente para la organización, lo que impide que en un futuro

Scire. $8: 2$ (jul.-dic. 2002) 159-172. 
puedan conectarse en red todas estas bases de datos; c) la difícil accesibilidad por parte de usuarios externos, caso de los investigadores, a los fondos conservados en estos medios; d) el escaso intercambio de información entre todas las organizaciones pertenecientes al mismo ámbito audiovisual andaluz. Este hermetismo del que hablamos no es constatable, por otro lado, en la esfera universitaria, ya que, primero, el tratamiento documental es muy similar en las diversas instituciones al guiarse todas ellas por las normas nacionales de descripción externa y de catalogación; segundo, la mayoría de los centros facilita la consulta de sus fondos a todos los usuarios externos necesitados de información contenida en su colección; y, tercero, en líneas generales, las universidades andaluzas analizadas mantienen entre ellas una política activa de relaciones interbibliotecarias.

Aunque los datos obtenidos en nuestra investigación puedan parecer desalentadores, deben en realidad servirnos, a quienes cultivamos la Documentación Audiovisual, de acicate o revulsivo para abrirnos al mundo exterior, mundo en el que debemos hacer uso de nuestros aprendizajes y conocimientos especializados. Cambiar la situación por la que actualmente atraviesa la práctica documental en los medios audiovisuales andaluces puede estar en nuestras manos, si todos, documentalistas y documentólogos andaluces provenientes de cualquier sector (de Ciencias de la Información o de Archivística, Biblioteconomía y Documentación), nos ponemos de acuerdo para emprender serias campañas informativas a través de las cuales demos a conocer a todas las organizaciones audiovisuales de nuestra Comunidad Autónoma qué es un servicio de documentación, cuáles son sus objetivos y funciones, y cómo este servicio ayudaría grandemente a mejorar la calidad de los programas producidos por nuestras empresas o instituciones, haciéndolas, por derivación, más competitivas en el mercado y en el marco global de la sociedad de la información, o más propiamente dicho, de la saturación informativa.

\section{Notas}

(1) Dicho trabajo de campo ha sido posible gracias al esfuerzo y colaboración de las siguientes personas: el director del Grupo de Investigación en Comunicación Científica y Desarrollo, Dr. Antonio García Gutiérrez, Catedrático de Periodismo de la Universidad de Sevilla, las coordinadoras del proyecto, Dra. Ángeles López Hernández, Profesora Asociada del Departamento de Periodismo de la Universidad de Sevilla, y M M $^{\mathrm{a}}$ Dolores Rodríguez Brito, Directora de la Biblioteca de la Facultad de Ciencias de la Información de la Universidad de Sevilla, junto a un equipo de alumnos de segundo ciclo de las licenciaturas de Periodismo, Comunicación Audiovisual y Publicidad y Relaciones Públicas de la Universidad de Sevilla formado por Carmen Cabello de Alba Fernández, Leonor Fernández Vargas, Anna García Sans, Tania González Redondo, Antonio J. Rodríguez Álvarez, Carmen Rodríguez Mendoza y José Enrique de la Rosa.

(2) Dicha base de datos se puede consultar en el Departamento de Periodismo de la Universidad de Sevilla. 